\title{
Chronic Obstructive Pulmonary Disease-A Disease of Accelerated Aging
}

\author{
An Expert Interview with Don D Sin
}

Division of Respiratory Medicine and Centre for Heart Lung Innovation, St Paul's Hospital, University of British Columbia, Vancouver, BC, Canada

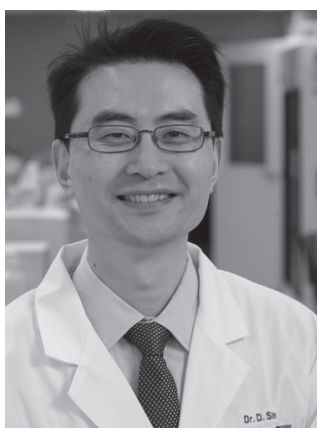

DOI: https://doi.org/10.17925/USRPD.2018.13.1.15

Don D Sin

Don Sin is Professor of Medicine at the University of British Columbia (UBC) and Director of the Centre for Heart Lung Innovation (HLI) at St. Paul's Hospital in Vancouver, Canada. He holds a Tier 1 Canada Research Chair in Chronic Obstructive Pulmonary Disease (COPD) and the De Lazzari Family Endowed Chair at HLI. He has published over 450 papers on COPD. He is currently the Section Editor of COPD for the European Respiratory Journal and an Associate Editor of the Canadian Journal of Respiratory, Critical Care and Sleep Medicine. He is also a special advisor to BMJ and sits on the editorial boards of the American Journal of Respiratory and Critical Care Medicine, Thorax and CHEST. He has been a member of the scientific committee of GOLD (Global Initiative for Chronic Obstructive Pulmonary Disease) since 2009. His research focus is using "omics" data to discover novel biomarkers of disease activity and new therapeutic targets to reduce hospitalization and mortality in patients with COPD.

\section{Keywords}

Aging, azithromycin, biomarker, COPD, exacerbation, mortality, quality of life, senescence, telomere

Disclosure: Don D Sin has received research funding from drug companies: AstraZeneca, Merck and Boehringer Ingelheim, and has received honoraria for serving on COPD advisory committees of Boehringer Ingelheim, AstraZeneca and Sanofi-Aventis.

Review Process: This is an expert interview and, as such, has not undergone the journal's standard peer review process.

Acknowledgment: Medical writing assistance was provided by Katrina Mountfort of Touch Medical Media and was supported by Touch Medical Media.

Authorship: The named author meets the International Committee of Medical Journal Editors (ICMJE) criteria for authorship of this manuscript, takes responsibility for the integrity of the work as a whole, and has given final approval for the version to be published.

open Access: This article is published under the Creative Commons Attribution Non-commercial License, which permits any non-commercial use, distribution, adaptation, and reproduction provided the original author and source are given appropriate credit. @ The Author 2018.

Received: July 15, 2018

Published Online: September 17, 2018

Citation: US Respiratory \& Pulmonary

Diseases. 2018;3(1):15-6

Corresponding Author: Don D Sin, MD, FCCP, Centre for Heart Lung Innovation, St. Paul's Hospital, 1081 Burrard St, Vancouver, BC, Canada, V6Z 1Y6.

E:Don.Sin@hli.ubc.ca Twitter: @donsin4

Support: No funding was received in the publication of this article.
C hronic obstructive pulmonary disease (COPD) is a major cause of morbidity and mortality throughout the world. COPD frequently coexists with other age-related co-morbidities, ${ }^{1}$ and is now known to be a disease of accelerated aging. ${ }^{2}$ The length of telomeres, which become progressively shorter as cells divide, has been proposed as a biomarker of aging. ${ }^{3}$ Shorter telomere length may therefore represent a useful biomarker for identifying COPD patients at increased risk for accelerated disease progression and poor outcomes. ${ }^{4}$ In an expert interview, Dr sin discusses his recently published study investigating the relationship of telomere length of leukocytes and clinical outcomes, including health status, rate of exacerbations, and risk of mortality in individuals with COPD. ${ }^{5}$

\section{Q. What is already known about the impact of COPD on accelerated aging?}

COPD is almost unheard of below 40 years of age. The risk of COPD increases exponentially once individuals pass this age threshold, such that above age 70 years, one in three septuagenarians and beyond have COPD. ${ }^{6}$ Because COPD causes accelerated aging, patients develop multiple age-related comorbidities such as heart disease, stroke, cancer and osteoporosis."

\section{Q. Could you tell us a little about your recent study investigating the utility of measuring telomere length in patients with COPD?}

We tested the hypothesis that telomeres, which are easily measured in blood, are biomarkers (or surrogates) of aging in COPD and most importantly whether telomeres are associated with poor outcomes for COPD patients. ${ }^{5}$ We measured the telomere length of DNA extracted from blood samples taken from 576 patients with moderate-to-severe COPD who were participants in the Macrolide Azithromycin for Prevention of Exacerbations of COPD (MACRO) study (ClinicalTrials.gov: NCT00325897). These patients were treated with azithromycin or placebo for 12 months and all were followed initially for approximately 12 months, during which time their overall health status and COPD exacerbations were recorded. They were then followed for a further 29 months to assess mortality. ${ }^{5}$

\section{Q. What were the findings of this study?}

We found that even though the average age of the patients in our study was 66 years, their biological age based on telomeres was 15-20 years older (in their 80s). We also found that patients with short 
telomeres, and thus of older biological age, were more likely to experience exacerbations and nine times more likely to die than those who had long telomeres (thus younger biological age). ${ }^{5}$

\section{Q. What were the effects of azithromycin treatment on COPD patients with short telomeres?}

The good news was that those patients who took azithromycin were protected from developing exacerbations when they had short telomeres. ${ }^{5}$

\section{Q. What is likely to be the impact of these findings on COPD treatment and what further studies are planned?}

We need to see whether these findings can be replicated in other settings. If we can reproduce these findings, we may be able to use blood telomeres, which are easy to measure as a simple blood test, to identify patients at high risk (biologically older) and place them on azithromycin to protect them against exacerbations. $\square$

Barnes PJ, Celli BR. Systemic manifestations and comorbidities of COPD. Eur Respir J. 2009;33:1165-85.

Ito K, Barnes PJ. COPD as a disease of accelerated lung aging. CHEST. 2009;135:173-80.

Mather KA, Jorm AF, Parslow RA, et al. Is telomere length a biomarker of aging? A review. J Gerontol A Biol Sci Med Sci. 2011;66:202-13.

Lee J, Sandford AJ, Connett JE, et al. The relationship between telomere length and mortality in chronic obstructive pulmonary disease (COPD). PLOS One. 2012;7:e35567.

Jin M, Lee EC, Ra SW, et al. Relationship of absolute telomere length with quality of life, exacerbations, and mortality in COPD. CHEST. 2018:154:266-73.

Pelkonen M, Notkola IL, Nissinen A, et al. Thirty-year cumulative incidence of chronic bronchitis and COPD in relation to 30-year pulmonary function and 40-year mortality: a follow-up in middle-aged rural men. CHEST. 2006;130:1129-37. 